\title{
Psychosocial Safety Climate Predicts Health and Safety Status of Ghanaian Fuel Attendants
}

\author{
Edward W. Ansah", Joseph K. Mintah, Joseph K. Ogah \\ Department of Health, Physical Education and Recreation (HPER), University of Cape Coast, Cape Coast, Ghana
}

Copyright $(2018$ by authors, all rights reserved. Authors agree that this article remains permanently open access under the terms of the Creative Commons Attribution License 4.0 International License

\begin{abstract}
The purpose of this study was to determine the level of psychosocial safety climate (PSC) at fuel stations in Accra and test the paths via which PSC predicts health and safety of the attendants. We surveyed 876 conveniently sampled attendants from four major Oil Marketing Companies (OMCs). A questionnaire derived from three pre-existing ones (PSC-12, Job Content Questionnaire and Short Form Health Survey) was used for data collection. It yielded composite reliability between 0.91 and 0.95. Partial Least Squared-Structural Equation Model was used for analysis. Results shows a high risk of (36.08) PSC perception among the attendants. PSC directly, and indirectly, via the path of job resources, predict health and safety of the attendants. Job demands have significant direct influence on health and safety with its path being the strongest $(r=-0.66)$ and most significant $(t=39.48, p=$ $0.00)$ compared with PSC ( $r=0.16)$ and job resources $(r=$ -0.12). The health, safety and well-being of the fuel station attendants appears to be preserved where management takes practical steps to raise PSC of the fuel stations and supervisor provides increased support for the attendants. An increase in PSC is most likely to cause increase in supervisor support to the attendants. In such stations, daily productivity is more likely to increase due to well-being of the workers.
\end{abstract}

Keywords Psychosocial Safety Climate, Job Demands, Job Resources, Safety Perception, Fuel Attendants

\section{Introduction}

Several researchers attempted to understand the workplace factors that are having effects on the health and safety of the workers [1, 2, 3, 4]. However, until recently [3, 5], the majority of these studies have focused on the workplace machinery and behaviours of the workers $[3,6]$. Furthermore, many of these studies linked workers' health and safety to the provision of safety devices, personal protective equipment (PPE), safety training and education [7], safety policy enforcement and availability of safety committee and unions. These studies placed the health and safety solely in the hands of workers, a belief that near misses, accidents resulting to injuries, illnesses or deaths are the result of the worker and/or physical structure at the workplace. However, organisational climate perception has been acknowledged as a critical component in determining employee health and safety. In addition, safety culture and climate researchers also lack the consensus and clarity about domains important in a culture of safety and how to score improvements over time [8]. For example, the relationship between safety climate and clinical outcomes are unclear. Further, measurement tools to score or improve workplace safety climate are also inconsistent (4, 7). It is also not clear how organisations can assess the influence of safety climate without placing safety behaviour of workers at the center. Therefore, the theory of psychosocial safety climate (PSC) and its studies have emerged as optimal alternative [3, 6].

The theory of PSC states that workers' health and safety can be determined when the organisational PSC is known [6]. PSC refers to a climate for employee psychological health and safety. It encompasses the level of senior management commitment and support for stress prevention; priority management gives to health and safety versus productivity goals; organisational communication, upwards and downwards in relation to health and safety matters; and the extent of participation and involvement by managers and workers in relation to health and safety [3]. PSC is a "shared perceptions of organisational policies, practices and procedures for the protection of worker psychological health and safety" (p. 1). This relates workplace health and safety largely to management safety practices [9] and makes workers participants and not objects in issues of workplace health and safety. A recent study revealed that as workplace health and safety scores reduce, employees face greater risks of depression and job stress [10]. Moreover, reduced workplace safety score is a demonstration of high job demands, less job control, increased injury and illness rates [11].

In a typical workplace environment, a high level of job demands leads to psychological distress of the workers 
[12]. However, this relationship could be offset when there are high job resources, influenced by the organisational context produced through senior managers' safety concerns. Thus, high levels of PSC is needed to promote efficient utilisation of job resources to reduce job demands outcomes. For instance, a longitudinal study conducted among Australian police officers revealed that increase PSC was necessary for resources to offset the influence of job demands on worker health outcomes such as depression and anxiety disorders [3]. These authors demonstrated that in the presence of high PSC, high emotional resources moderated the positive relationship between emotional demands and change in workgroup distress. Then, PSC becomes a preeminent stress reduction factor that mediates the interactions among job demands, resources and psychological health problems such as depression and anxiety among workers [12]. Similarly, a study [13] demonstrated, with Malaysian employees' data, that PSC was negatively related to job demands that in turn associated with burnout and cynicism. Using structural equation modeling's bootstrapping algorism, this cross-sectional data indicated that PSC was also related to job performance through a positive relationship with job resources and job satisfaction. In another Malaysia study, a mediating effect of PSC on job demands-depression relation was found [14]. Further, via the path of PSC, job resources either supervisor support or co-worker support had a positive relation with job satisfaction of the workers. In this case, organisational PSC becomes the hub, ladder and precedes many other critical factors in the workplace, and the worker health and safety interaction (see Figure 1).

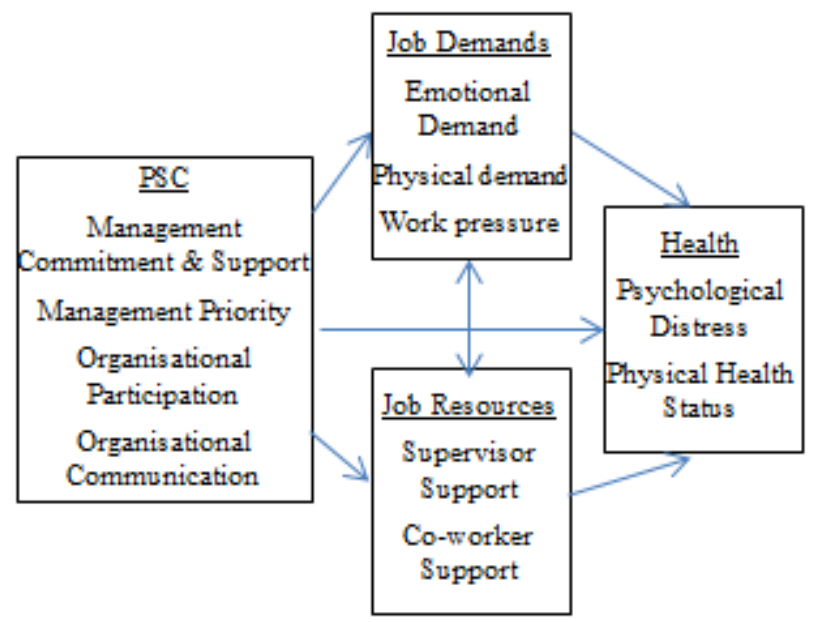

Figure 1. PSC, Job Demands, Job Resources and Worker Health and Safety Interaction

Health and safety literature is sparse from critical sectors like fuel stations and their attendants. This may be so because the western world does not normally use typical fuel attendants rather relied on self-served. Even in the developing world where attendants are used at the downstream oil marketing, health and safety literature is very limited. However, the discovery of oil in commercial quantity in Ghana has brought about the proliferation and setting up of fuel service stations all over the country especially in big cities like Accra, Tema, Kumasi and Takoradi [2]. This development is also promoting the rise in the employment numbers, with likely increases in the rate of physical and psychological work demands and their associated psychosocial health, safety and well-being challenges [6]. These workplace challenges are good grounds for occupational diseases, accidents, injuries and near misses [11] and the economic costs to the employees and employers [15]. For instance, Ghana is experiencing rampant fuel service station disasters in recent years. These accidents are the causes of many deaths including attendants and leaving many other workers and customers severely injured [2, 16]. Notable was the June 3rd 2015 "twin disaster", of fire and flood that killed about 159 lives including all the fuel attendants on duty [17]. The remote cause of this particular disaster was fuel tank leakage, which occurs in most fuel stations during delivery of fuel into underground tanks. Besides, evidence suggests that the fuels sold by these attendants in Ghana's market contain high amount of sulphur (3000 ppm) and other volatile organic compounds [18]. These compounds have both acute and/or chronic debilitating health effects on the workers, including low and defective sperm [19], lead and benzene poisoning [20], concentration problem, pregnancy defects, cardio-respiratory defects and cancers [21]. Therefore, research evidence and protecting these categories of workers is of greater concern now than ever. But only two studies have attempted studying health and safety of fuel attendants in Ghana [2, 16]. An attempt to investigate the relationship between safety measures of oil marketing companies (OMCs) and safety behaviours of the attendants found a very low provision of measures like PPE by the companies [2]. These authors concluded that availability of adequate safety measures was important to increase safety behaviours among the attendants. Workers' safety behaviour is key to protecting their health and that of others associated with the work environment [2]. Another that study explored health and safety standards, occupational hazards and health problems among attendants also concluded that attendants in Kumasi are predisposed to "dire health risks due to their working conditions and require urgent measures" [16. p. 139] to protect them. Though, a good attempt, these two studies concentrated on attendants in Central and Western regions and Kumasi in Ashanti region. The results and conclusions of these studies are limited and do not represent the vast majority of attendants and their companies in Ghana. Moreover, they failed to address the fundamental issues, such as PSC, confronting workplace health and safety in the developing nations in contemporary times.

Though health and safety literature is increasing with PSC studies in the developed nations [1, 22], they focused predominantly on psychological health [23], to the neglect of physical health and safety of the workers. Furthermore, in sub-Saharan African there is limited available research 
evidence on the theory of PSC, its related constructs and their influence on the physical health and safety of workers. Also, in many organisations or population segments including fuel stations attendants, PSC and its closely related variables have not yet been studied. Therefore, the aim of this study was to determine the level of PSC at fuel service stations in Accra and test the extent and the paths through which PSC, job resources and job demands predict health and safety of the attendants.

\section{Materials and Methods}

\subsection{Participants' Selection}

We employed quantitative survey to study 876 conveniently sampled fuel station attendants. These attendants were selected from four major OMCs in Accra, Ghana. These OMCs control the biggest market shares in Ghana's downstream oil industry. Perhaps, they also have long standing experiences in their operations both in Ghana and outside the country [2]. The attendants included 56\% $(n=492)$ males and $44 \%(n=384)$ females, age ranged 15 to 66 years $(M=28, S D=6.5)$. They also comprised $49 \%$ $(n=426)$ forecourt attendants, $28 \%(n=245)$ shop attendants and 23\% $(n=205)$ lube bay mechanics. Thirteen percent $(n=115)$ of the participants had basic education or below, $10 \%(n=83)$ vocational, $67 \%(n=588)$ secondary and $10 \%(n=88)$ tertiary. For their health and safety status, $16 \%(n=136)$ reported it either poor or very poor, $21 \%(n$ $=181)$ fair (not poor and not good), 37\% $(n=325)$ good and 26\% $(n=234)$ very good or excellent.

\subsection{Instruments}

We used three pre-existing instruments; PSC-12 [3, 5], Job Content Questionnaire [24] and Short Form Health Survey [19] to collect data from the participants. PSC-12 instrument [6] focuses on four specific segments; (1) senior management commitment and involvement in relation to stress prevention practices, (2) management priority given to psychological health and safety in comparison to productivity goals, as perceived by employees, (3) organisational communication measures workers' views of feedback on well-being and (4) organisational participation that emphasis the consultation regarding health and safety issues with employees and their unions. Responses were made on a 4-point Likert scale from 1 (strongly disagree) to 4 (strongly agree). The scores for each of the 12 items from all subscales were aggregated to produce the overall score ranging from a minimum 12 to maximum score of 60 that measured the overall PSC level.

Job Demands and job resources items were taken from the Job Content Questionnaire [24]. Job demands items included the physical demands (four items), work pressure (four items), and emotional demands (four items) subscales. Participants responded to the items on a 4-point Likert scale from 1 (strongly disagree) to 4 (strongly agree).
Meanwhile, job resources measured both supervisor ('My supervisor is successful in getting people to work together') and co-worker support ('People I work with take a personal interest in me') with 4 items each. Responses ranged from 1 (strongly disagree) to 4 (strongly agree).

Physical health and safety of the participants was measured using 8 items from the Short Form Health Survey [25]. The instrument measures participants' physical health status over the previous 4 weeks interval; 'During the past 4 weeks, how much did physical health problems limit your usual physical activities other than work?', 'During the past 4 weeks, how much did your physical health problems limit your usual social activities with family or friends?' All responses are on 5 points scale.

The pre-existing instruments have been validated across nations and worker segments and recorded high and acceptable psychometric prosperities [26, 27, 28, 29, 30]. Our data also yielded high composite reliability values; PSC $=0.95$, job demands $=0.95$, health and safety $=0.92$, and job resources $=0.91$. Also, indicators loaded highest on their constructs and recorded reliability values between 0.56 and 0.87 , higher than the cutoff point of 0.50 [31]. The questionnaire further yielded high and acceptable convergent validity values; PSC $=0.65$, job demands $=$ 0.76 , job resources $=0.72$ and health and safety $=0.66[32$, 33].

The questionnaire was structured in three parts; one, two and three. Part one collected participants' demographic information such as age, gender, education and department they work at the fuel stations (10 items). Part two included 8 items which solicited information on health and safety of the attendants. Part three measured PSC, job demands and job resources.

\subsection{Procedures}

The University Institutional Review Board (IRB) granted the ethical approval for this study. Also, we sought and obtained authorization from the various company managements and the managers of each fuel station. The attendants were contacted at the stations through their station managers and supervisors who in most cases assisted in the distribution of the instrument. Attendants filled the questionnaire after the purpose has been thoroughly explained to them. They were given three days to return the completed questionnaire since most of the fuel stations run 24-hour shift. The participants were assured of their anonymity, confidentiality and voluntary participation and that they can stop answering the questionnaire in the process. Furthermore, each participants signed an informed consent form before taken part in the study.

\subsection{Data Analyses}

The level of PSC at fuel stations was calculated by aggregating the PSC-12 items, based on Sobel [34] 
benchmark. A mean score of $\geq 41$ indicates a high PSC (protective to health and safety), between 37.99 and 40.99, moderate risk and $\leq 36.99$, low PSC (very high risk). In addition, we performed PLS-SEM analysis [35], to assess the research models. We modeled PSC, job demands and job resources as exogenous latent variables with health and safety as endogenous. PSC was directly and indirectly, via the paths of job demands and job resources modeled on health and safety of the attendants. The data satisfied all the necessary validity and reliability criteria such as composite reliability, convergent and discriminant validity. For example, constructs recorded composite reliability of 0.70 or higher. In addition, the indicators and constructs' average variance extracted (AVE) of 0.60 or higher were acceptable [33, 35, 36] (see Table 1 and 2).

\section{Results}

The aim of this study was to measure the level of PSC and explore the paths via which it influences the health and safety status of fuel station attendants. The results revealed an average PSC mean score of 36.08 at the fuel stations surveyed. This is a high risk perceived organisational PSC [6]. There is high propensity of workers being exposed to both physical and psychological health and safety challenges such as high rate of injuries and illnesses in such organisations. Further analysis using PLS-SEM indicated that the model achieved a good fit, SRMR of 0.06 (criterion $\leq 0.08$ ) with iteration at 7 . The model also satisfied item and construct reliability requirements [32, 35]. The indicator outer loadings and cross-loadings, for retained items, were between 0.74 and 0.86 on PSC, 0.83 and 0.90 on job demands, 0.81 and 0.89 on job resources, and 0.73 and 0.90 on health and safety. Besides, the variables' composite reliability are between 0.91 and $0.95,0.70$ cutoff point. Furthermore, inner model collinearity evaluation indicated that the constructs are distinct and had no problem with multicollinearity. These were calculated for only the exogenous latent variables, at significant $p=0.05$, with tolerance values less than 1.0 and VIF more than 1.0 [33]. The VIF values for PSC, job demands and job resources are 1.16, 1.00 and 1.16, respectively (see Table 1). The latent variables, PSC, job demands, job resources and health and safety also achieved acceptable discriminant validity [36, 37] (see Table 2). However, indicator loadings on the initial analysis revealed that some items loaded poorly (below 0.70) and therefore were removed [37]. For instance, Heal3 and Heal6 under Health and Safety; Job Demands3, Job Demands5, Job Demands6, Job Demands7, Job Demands9 and Job Demands12 under the Job Demands construct; Job Resources 5 to 8 and PSC4 indicators were removed before further analysis.

Table 1. Summary of the Results of Reflective Model and Multicollinearity Diagnostic

\begin{tabular}{|c|c|c|c|c|c|c|}
\hline \multirow{2}{*}{ Latent Variables (LV) } & \multirow{2}{*}{ Indicators } & \multirow{2}{*}{ Loadings } & \multirow{2}{*}{ Composite Reliability } & \multirow[t]{2}{*}{ AVE } & \multicolumn{2}{|c|}{ Collinearity Statistics } \\
\hline & & & & & Tolerance & VIF \\
\hline Health and Safety & & & 0.919 & 0.655 & & \\
\hline & $\begin{array}{l}\text { Heal1 } \\
\text { Heal2 } \\
\text { Heal4 } \\
\text { Heal5 } \\
\text { Heal7 } \\
\text { Heal8 }\end{array}$ & $\begin{array}{l}0.776 \\
0.838 \\
0.895 \\
0.728 \\
0.886 \\
0.752\end{array}$ & & & & \\
\hline Job Demands & & & 0.949 & 0.755 & 0.99 & 1.02 \\
\hline & $\begin{array}{c}\text { Job Demands1 } \\
\text { Job Demands2 } \\
\text { Job Demands4 } \\
\text { Job Demands8 } \\
\text { Job Demands10 } \\
\text { Job Demands11 }\end{array}$ & $\begin{array}{l}0.828 \\
0.894 \\
0.901 \\
0.851 \\
0.863 \\
0.876\end{array}$ & & & & \\
\hline Job Resources & & & 0.913 & 0.724 & 0.86 & 1.16 \\
\hline & $\begin{array}{l}\text { Job Resources1 } \\
\text { Job Resources2 } \\
\text { Job Resources3 } \\
\text { Job Resources4 }\end{array}$ & $\begin{array}{l}0.870 \\
0.889 \\
0.831 \\
0.811 \\
\end{array}$ & & & & \\
\hline PSC & & & 0.953 & 0.650 & 0.86 & 1.16 \\
\hline & $\begin{array}{l}\text { PSC1 } \\
\text { PSC2 } \\
\text { PSC3 } \\
\text { PSC5 } \\
\text { PSC6 } \\
\text { PSC7 } \\
\text { PSC8 } \\
\text { PSC9 } \\
\text { PSC10 } \\
\text { PSC11 } \\
\text { PSC12 }\end{array}$ & $\begin{array}{l}0.767 \\
0.737 \\
0.802 \\
0.817 \\
0.857 \\
0.826 \\
0.806 \\
0.834 \\
0.838 \\
0.833 \\
0.749\end{array}$ & & & & \\
\hline
\end{tabular}


Table 2. Discriminant Validity using Fornell and Larcker Criterion

\begin{tabular}{|c|c|c|c|c|}
\hline Latent Variables & $\mathrm{H} \& \mathrm{~S}$ & JD & JR & PSC \\
\hline Health \& Safety (H\&S) & 0.810 & & & \\
\hline Job Demands (JD) & 0.671 & 0.869 & & \\
\hline Job Resources (JR) & 0.040 & 0.008 & 0.851 & \\
\hline Psychosocial Safety Climate (PSC) & 0.148 & 0.073 & 0.481 & 0.806 \\
\hline
\end{tabular}

In further analysis, combined PSC, job demands and job resources explained $47 \%$ of the variance in health and safety $\left(R^{2} a d j=0.47\right)$ with PSC alone explaining $23 \%$ of job resources $\left(R^{2} a d j=0.23\right)$. In addition, the model's path coefficients suggested that job demands has the strongest but negative $(r=-0.66)$ direct effect on health and safety, followed by PSC's positive effect $(r=0.16)$ and job resources $(r=-0.12)$. Besides, PSC has moderate effect on job resources $(r=0.48)$ but weak and non-significant, less than $0.1(r=0.07)$ on job demands [36] (see Figure 2).



Figure 2. PLS-SEM Reflective Model Predicting Health and Safety from PSC, Job Demands and Job Resources

The PLS bootstrapping further confirmed the paths' strength and significance except for that between PSC and job demands. The figures on the hypothetical paths in the model indicate the significance and relevance of each construct to the other in the model. In addition, the values between the indicators and their latent variables reveal relevance and significance contribution of each indicator to its construct in the model, and the higher such values the better. Thus, any value below or equal to 1.96 at 0.05 , indicates an irrelevant and non-significant path [38]. In that case, PSC becomes a direct and significant predictor of health and safety (5.626) and job resources (18.895) but not on job demands (1.943). Moreover, both job demands
(39.484) and job resources (4.254) are direct, relevant, and significant predictors of health and safety, with job demands the strongest. Indirectly, via the path of job resources, PSC significantly and relevantly predicted health and safety of the attendants. The conceptual model reproduced Q-square values between 0.16 and 0.29 and indicator cross-validated redundancy (q-square) between 0.12 and 0.52 . This indicates the relevance and significance of the overall model containing PSC, job demands and job resources in predicting health and safety of the fuel station attendants [39] (see Figure 3). Therefore, PSC, job demands and job resources are significant and relevant direct predictors of health and safety of the attendants. 


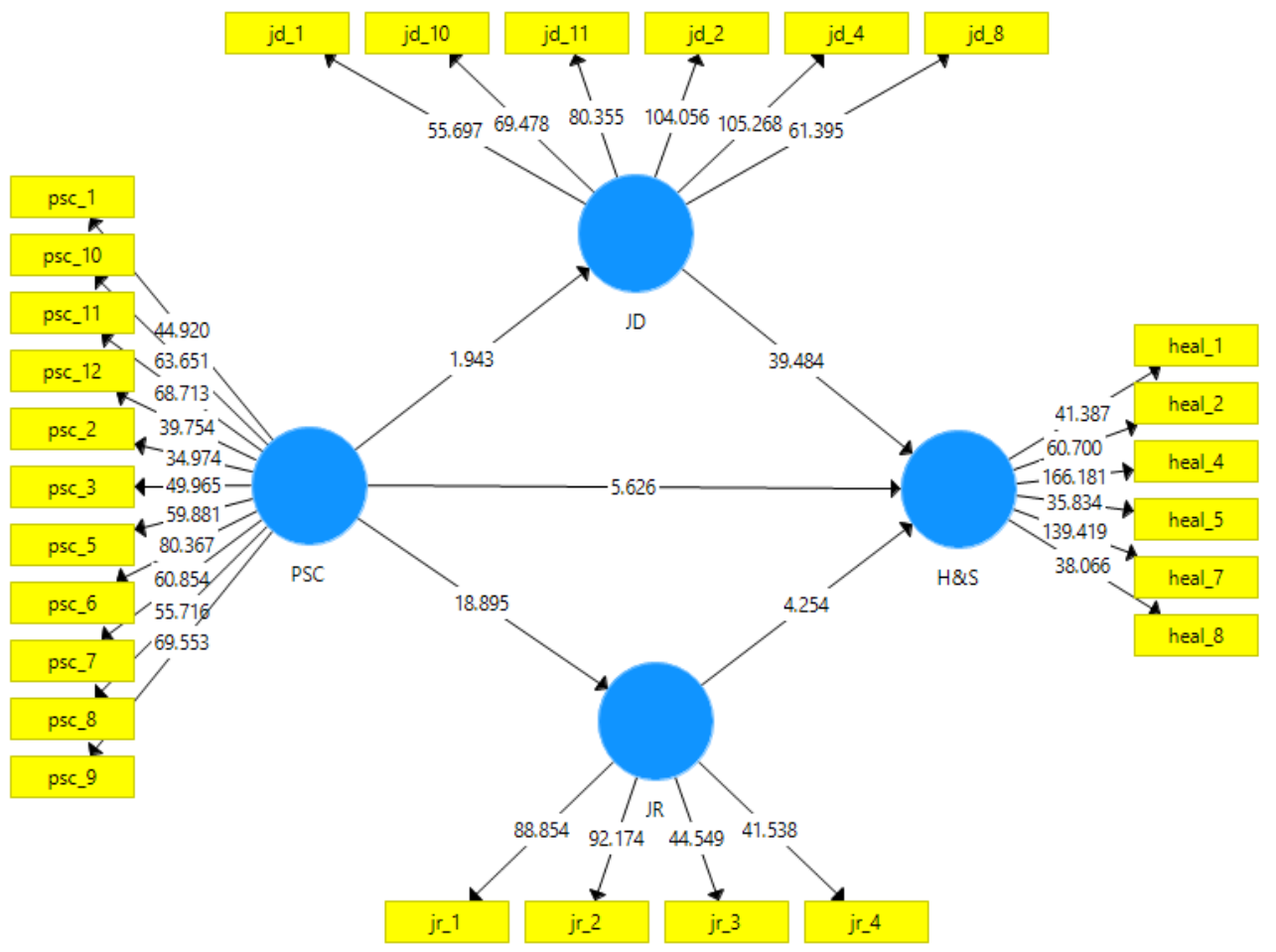

Figure 3. Predicting Health and Safety from PSC, Job Demands and Job Resources Bootstrapping with Procedure

\section{Discussion}

The study aimed at determining the level of PSC at the fuel stations and test the path(s) through which PSC predicts health and safety of the fuel station attendants in Accra. Attendants in this study perceived their stations to pose a high risk to their health and safety. A possible reason is that the OMCs/FSSs or a vast majority of them, represented by their senior officers, do not adequately provide supportive PSC environment or measures that aim at promoting the health and safety of the attendants $[5,6]$. However, evidence strongly suggests that a high perceived organisational PSC is a buffer against deleterious impacts of daily job demands. Such high level of daily job demands may lead to injuries and ill health of workers at their fuel stations [40]. And that, where workers' perception about the workplace PSC is high, they also have better and improved physical and psychological health. Further, such improved organisational PSC has a tendency to lessen the burden of emotional job demands among workers [6, 28]. Hence, every attempt must be made to rise the PSC of these fuel stations to give importance to the health and safety needs of the attendants.

The findings also indicated that PSC, job demands and job resources are relevant predictors of health and safety of the attendants. Distinctively, PSC and job demands are significant, and moderate predictors of health and safety whereas job resources is a significant, but a low predictor of attendants' health and safety state. Furthermore, PSC indirectly via the path of job resources, predicts the health and safety of these attendants. The non-significant path of
PSC $\rightarrow$ job demands to health and safety indicates a low level of PSC and high perceived job demands on the attendants. Furthermore, the negatively low relationship between job resources and health and safety confirms the non-existence or low level of job resources (supervisor and co-worker support) as experienced by the attendants. These findings confirm the assertion of many researchers that work climate, whether safety or economic, affects both, directly and indirectly, the health, safety and well-being of the typical worker [5, 41].

It is important to make a point that PSC, job demands and job resources exist together in every workplace. Perhaps, the degree to which they exist and influence each other is the matter of concern to health and safety researchers and managers. In the first instance, PSC is a "cause of cause" [5, p. 2], an antecedent factors aiming at health, safety and the well-being of workers [11]. At the fuel station, PSC would be demonstrated by the extent to which senior managers show commitment and are supportive to preventing situations deem stressful to the attendants. The OMCs and their station managers could do this by prioritizing the health and safety needs of the attendants over rising in daily sales [42], a goal which is normally achieved at the back of increased health and safety [43]. Furthermore, protective PSC is seen in the level and channel of management's health and safety communication, the extent of their participation and involvement about safety issues at these fuel stations. In that case, managers' ability to bring on board attendants in matters relating to health and safety would enhance attendants' personal responsibility towards self and 
organisational protection [38]. Law et al. [9] view PSC as a shared perception, therefore, it is believed to directly and indirectly influence the protection of the workers' health and safety [26]. For example, PSC is effective in promoting the health and safety of workers where it was found to lower chronic fatigue and improved work engagement [44], and reduce depression and fatigue [45]. Thus, low recorded PSC at the fuel station is exposing the attendants to higher degrees of job pressure, a situation which leads to psychological and physical adverse health conditions.

Furthermore, finding revealed that whereas PSC indirectly, via the path of job resources, influences health and safety, job demands and job resources have direct effects on attendants' health and safety. This goes to confirm the low level of PSC at OMCs and fuel service stations. In such a workplace, workers' health and safety status are going to be compromised while they experience the burden of job demands [46, 47]. To support this assertion, Dollard and Bakker [6] indicated among a number of Australian workers that the main effect of PSC on psychological health problems became non-significant after controlling for job demands. In a large diverse sample from the developed world as Australia, it is expected that the workers would acknowledge the effects of job demands on their health and safety. Moreover, where PSC is lower, as reported PSC in this case, the effects of job demands on the health and well-being of workers become prominent [40]. However, despite the fact that the current study uses the same kind of analytical tool (SEM-PLS) as the previous one, the attendants are from one industrial sector and their job demands are high and monotonous. We argued then that in the presence of the chronic job demands, attendants experience injuries, psychological distress, emotional exhaustion and other physical health problems [48]. On the other hand, where attendants experience the actualization of higher PSC, supervisors' would increase support for the attendants who in turn increase support for each other. The result would be a reduced effects of job demands on their health, safety and well-being of the attendants. This is more likely to result in improved physical and psychological health and safety [4] among the attendants. But this seems not to be the case among participants in this current research.

The indirect path of PSC through job resources further indicates the antecedence nature of PSC to other workplace health and safety promoting variables such as job resources [23, 28]. Findings of other studies indicated that job resources moderate the influence of PSC on health and safety among various worker groups [6, 40]. For instance, a meta-analysis [49] showed that the health erosion and motivational pathways are mechanisms by which resources relate to health. Accordingly, PSC is expected to precipitate these paths and prevent errors, accidents, injuries and deaths resulting from the workplace [44]. Both PSC and job resources are organisational variables that aim to protect, and promote the health, and safety of the worker $[3,5,14]$. Thus, at a typical OMC or fuel service station, the safety management practices [2], which reflect PSC, has direct effects on how job resources are manifested and perceived by fuel attendants $[9,50]$. Therefore, it becomes the responsibility of the senior management including sales executives, area heads, marketing managers, accountants, auditors, and station managers to promote PSC and increase the supervisor support at the various fuel stations. In such OMCs and fuel stations, attendants are also likely to provide support to each other, an act that is necessary to reduce the effects of job demands on health and safety of the workers [13, 47]. For example, supervisors are importance at the fuel station because they are the immediate line management staff that liaise between the senior management and interact regularly with the workers [2]. The effectiveness of this daily supervisor-attendant interaction is supposed to result in positive attendant-attendant work relationship [50]. In such instance, supervisor-worker and worker-worker relationship has promotive effects on health, safety and well-being of the employees [45]. It implies further that at fuel stations where there is a high positive safety climate, supervisors are more likely to provide both physical and emotional assistance to the fuel attendants.

\section{Conclusions}

It is important to note that PSC is a policy-driven organisational variable within which other policies, procedures and practices are created, the aim is to increase the health and safety of the worker. Thus, both national and organisational level safety policies are important to reaching the health and safety goals of attendants. Within PSC, it is prudent that senior management makes effort to formulate policies that regulate their own safety activities. This safety policy should aim at giving priority to attendants' safety compared to only increasing sales. Further, there should be conscious effect towards promoting of bottom-up safety communication where it is likely attendants would own decisions and take appropriate actions to affect them. Senior management needs to actively take part in all matters concerning health and safety issues at the fuel stations. They need to proactively train attendants in health and safety promotion and bring them on board in matters of safety. It is equally adequate to task sales executives and marketing managers of the OMCs and managers of fuel service stations to make effective policies and regulations that aim at enhancing the health and safety of the fuel station attendants. Fuel station policies have to promote effective worker cooperation including supervisor-worker interaction. There should always be plans to monitor PSC and prevent stress among the attendants.

Though this is the first ever research attempt into the 
psychosocial safety factors influencing the health and safety of fuel station attendants in Ghana and maybe sub-Saharan African, it still has some limitations. The results of this study may underestimate the level of PSC at the fuel stations since only attendants from four major companies were involved. Besides, the convenient sampling nature of the participants could limit the generalization of the findings and conclusions. Nevertheless, the strong statistics provides for the validity and the reliability of the findings and conclusions. Since attendants' health and safety is a product of changes in workplace key factors, there is the need to explore such change as PSC, job demands and job resources change over time.

\section{REFERENCES}

[1] N. H. B. Abd Radzaz, and Bahari, S. F. "Psychosocial safety climate in organization: An overview of theoretical and empirical development”, Journal of Social and Development Sciences, vol. 4, no. 9, pp. 407-411, 2013.

[2] E. W. Ansah, and J. K. Mintah, "Safety management practices at fuel service stations in Central and Western Regions of Ghana”, Nigerian Journal of Health Education, vol. 16, no. 1, pp. 78-89, 2012.

[3] M. F. Dollard, T. Bailey, S. McLinton, P. Richards, W. McTernan, A. Taylor, and S. Bond, "The Australian Workplace Barometer: Report on psychosocial safety climate and worker health in Australia”, Magill Campus: Centre for Applied Psychological Research, 2012.

[4] P. U. Okoye, and Y. W. Aderibigbe, "Comparative assessment of safety climate of casual and permanent construction workers in South-East Nigeria. International Journal of Health and Psychology Research, vol. 2, no. 1, pp. 54-66, 2014.

[5] M. F. Dollard, T. Opie, S. Lenthall, J. Wakerman, S. Knight, S. Dunn, ... M. MacLeod, "Psychosocial safety climate as an antecedent of work characteristics and psychological strain: A multilevel model”, Work \& Stress: An International Journal of Work, Health \& Organization, vol. 26, no. 4, pp. 385-404, 2012.

[6] M. F. Dollard, and A. B. Bakker, "Psychosocial safety climate as a precursor to conducive work environments, psychological health problems, and employee engagement”, Journal of Occupational Ergonomic and Psychology, vol. 83, pp. 579-599, 2010.

[7] J. R. Wilkins, “Construction workers' perceptions of health and safety training programmes" Journal of Construction Management and Economics, vol. 29, no. 10, pp. 1017-1026, 2011.

[8] P. Pronovost, and B. Sexton, "Assessing safety culture: Guidelines and recommendations”, Qual Saf Health Care, vol. 14, pp. 231-233, 2005.

[9] R. Law, M. F. Dollard, M. R. Tuckey, and C. Dormann, "Psychosocial safety climate as a lead indicator of workplace bullying and harassment, job resources, psychological health and employee engagement”, J Acc Anal \& Prev., vol. 43, no. 5, pp. 1782-1793, 2011.

[10] T. S. Bailey, M. F. Dollard, and P. A. M. Richards, “A national standard for psychosocial safety climate (PSC): PSC 41 as the benchmark for low risk of job strain and depressive symptoms", Journal of Occupational Health Psychology, vol. 20, no. 1, pp. 15-26, 2015.

[11] P. C. Winwood, R. Bowden, and F. Stevens, "Psychosocial safety climate: Role and significance in aged care”, Occp Med \& Heal Aff., vol. 1, no. 6, pp. 1-5, 2013.

[12] M. A. Idris, M. F. Dollard, J. Coward, and C. Dormann, "Psychosocial safety climate: Conceptual distinctiveness and effect on job demands and worker psychological health”, Safety Science, vol. 50, no. 1, pp. 19-28, 2012.

[13] M. A. Idris, M. F. Dollard, and A. H. Winefield, "Integrating psychosocial safety climate in the JD-R model: A study amongst Malaysian workers”, AS Journal of Industrial Psychology, vol. 37, dol:10.4102/saiip.v3712.851, 2011.

[14] M. A. Idris, and M. F. Dollard, "Psychosocial safety climate, work conditions, and emotions in the workplace: A Malaysian population-based work stress study", International Journal of Stress Management, vol. 18, no. 4, pp. 324-347, 2011.

[15] S. Tappura, M. Sievanen, J. Heikkla, A. Jussila, and N. Nenonen, "A management accounting perspective on safety”, Safety Science, vol. 70, pp. 1-9, 2014.

[16] I. Monney, J. B. Dramanni, A. Aruna, A. G. Tenkorang, and F. Osei-Poku, "Health and safety in high-risk work environment: A study of fuel service stations in Ghana”, Journal of Environmental \& Occupational Science, vol. 4, no. 3, pp. 132-141, 2015.

[17] E. Smith-Asante, "A year after the June 3 twin disaster", 2016. Retrieved July 19, 2016, from http://www.graphic.com.gh/features/features/a-year-after-th e-june-3-twin-disaster

[18] M. Gueniat, M. Harjono, A. Missbach, and G. V. Viredaz, "Dirty diesel. How Swiss traders flood Africa with toxic fuels”, Accra: Public Eye. pp. 2016.

[19] J. M. Moline, A. L. Golden, N. Bar-Chama, E. Smith, M. E. Rauch, E. R. Chapin, ... P. J. Landrigan, "Exposure to hazardous substances and male reproductive health: A research framework", Journal of Environmental Health Perspective, vol. 108, pp. 803-813, 2000.

[20] B. Onunkwor, O. Dosumu, O. O. Odukoya, T. Arowolo, and O. Ademuyiwa, "Biomarkers of lead exposure in petrol station attendants and auto-mechanics in Abeokuta, Nigeria: Effect of 2-week ascorbic acid supplementation”, Environmental Toxicology and Pharmacology, vol. 17, pp. 169-176, 2004.

[21] M. D. Attfield, P. L. Schleiff, J. H. Lubin, A. Blair, P. A. Stewart, R. Vermeulen, ... D. T. Silverman, "The diesel exhaust in miners study: A cohort mortality study with emphasis on lung cancer”, Journal of National Cancer Institute, vol. 104, pp. 1-15, 2012.

[22] K. M. Zinsser, and A. Zinsser, "Two case studies of preschool psychosocial safety climates”, Research in Human Development, vol. 4, pp. 49-64, 2016. 
[23] Yulita, M. A., Idris M. F., Dollard, "Psychosocial safety climate: Past, present, and future research", In A. Shimazu, R. B. Nordin, M. F. Dollard, J. Oakman (pp. 89-134). "Psychosocial factors at work in the Asia Pacific: From theory to practice”, Switzerland: Springer International Publishing, pp. 89-134, 2016.

[24] R. Karasek, C. Brisson, N. Kawakami, I. Houtman, P. Bongers, and B. Amick, (1998). The Job Content Questionnaire (JCQ): An instrument for internationally comparative assessments of psychosocial job characteristics. Journal of Occupational Health Psychology, vol. 3, pp. 322-355, 1998.

[25] E. Ware, M. Kosinski, J. E. Dewey, and B. Gandek, "How to score and interpret single-item health status measures: A manual for users of the SF-8 health survey”, Lincoln, RI: Quality Metric Inc, 2001.

[26] T. S. Bailey, M. F. Dollard, and P. A. M. Richards, “A national standard for psychosocial safety climate (PSC): PSC 41 as the benchmark for low risk of job strain and depressive symptoms", Journal of Occupational Health Psychology, vol. 20, no. 1, pp. 15-26, 2015.

[27] Choi, S. Ko, and P. O. Ostergren, "Validity test of the IPD-work consortium approach for creating comparable job strain groups between Job Content Questionnaire and demand-control questionnaire”, International Journal of Occupational Medicine and Environmental Health, vol. 28, no. 2, pp. 1-13, 2015.

[28] M. F. Dollard, P. Richards, W. McTernan, T. Bailey, B. Daniels, and S. McLinton, "Psychosocial risk \& it's impact in Australian workplaces: Results from the Australian Workplace Barometer", South Australia: Centre for Applied Psychological Research, Work \& Stress Research Group, 2012.

[29] A. Juarez-Garcia, A. Vera-Calzaretta, G. Blanco-Gomez, V. Gomez-Ortız, E. Hernandez-Mendoza, J. Jacinto-Ubillus, and B. Choi, "Validity of the effort/reward imbalance questionnaire in health professionals from six Latin-American countries', American Journal of Industrial Medicine, vol. 58, no. 6, pp. 636-649, 2015.

[30] G. H. Villalobos, A. M. Vargas, M. A. Rondon, and S. A. Felknor, "Validation of new psychosocial factors questionnaires. A Colombian national study", American Journal of Industrial Medicine, vol. 56, pp. 111-123, 2013.

[31] M. Sarstedt, J. F. Hair, C. M. Ringle, K. O. Thiele, and S. P. Gudergan, "Estimation issues with PLS and CBSEM: Where the bias lies", Journal of Business Research, vol. 69, pp. 3998-4010, 2016.

[32] J. F., Hair, M. Sarstedt, L. Hopkins, and V. G. Kuppelwieser, "Partial least squares structural equation modeling (PLS-SEM): An emerging tool in business research”, European Business Review, vol. 26, no. 2, pp. 106-121, 2014.

[33] G. D. Garson, "Partial least squares: Regression \& structural equation models”, Asheboro: G. David and Statistical Associates Publishing, 2016.

[34] M. E. Sobel, "Asymptotic confidence intervals for indirect effects in structural equation models”, In S. Leinhardt (Ed.), "Sociological methodology", (pp. 290-312). Washington DC: American Sociological Association, 1982.
[35] C. M. Ringle, S. Wende, and J. M. Becker, "SmartPLS 3", Boenningstedt: SmartPLS GmbH 2015.

[36] M. Ringle, and M. Sarstedt, "Gain more insight from your PLS-SEM results: The importance-performance map analysis”, Industrial Management \& Data Systems, vol. 116, no. 9, 1865-1886, 2016.

[37] G. Fornell, and D. F. Larcker, "Evaluating structural equation models with unobservable variables and measurement error”, Journal of Marketing Research, vol. 18, no. 1, pp. 39-50, 1981.

[38] J. Henseler, C. M. Ringle, and M. Sarstedt, “A new criterion for assessing discriminant validity in variance-based structural equation modeling”, Journal of the Acad. Mark Sci., vol. 43, 115-135, 2015.

[39] M. Sarstedt, C. M. Ringle, J. Henseler, and J. F. Hair, "On the emancipation of PLS-SEM”, Long Range Planning, vol. 47, no. 3, pp. 161-167, 2014.

[40] A. Garrick, A. S. Mak, S. Cathcart, P. C. Winwood, A. B. Bakker, and K. Lushington, "Psychosocial safety climate moderating the effects of daily job demands and recovery on fatigue and work engagement”, Journal of Occupational and Organizational Psychology, vol. 87, pp. 694-714, 2014.

[41] M. F. Dollard, A. Shimazu, R. B. Nordin, P. Brough, and M. R. Tuckey, (Ed) "Psychosocial factors at work in the Asia Pacific”, New York: Springer Science+Business, 2014.

[42] R. Kaynak, A. T. Toklu, M. Elci, and I. T. Toklu, "Effects of occupational health and safety practices on organizational commitment, work alienation, and job performance: Using the PLS-SEM approach”, International Journal of Business and Management, vol. 11, no. 5, pp. 146-166, 2016.

[43] H. Becher, M. F. Dollard, Asia Pacific Center for Work Health and Safety, WHO Collaborating Center in Occupational Health and University of South Australia. "Psychosocial safety climate and better productivity in Australian workplaces costs, productivity, presenteeism and absenteeism”, Australia: Safe Work Australia, 2016.

[44] M. F. Dollard, T. Opie, S. Lenthall, J. Wakerman, M. MacLeod, G. Rickard, and S. Knight, "A multilevel model of psychosocial safety climate as a lead indicator of job demands, control and support and psychological health", University of South Australia: Work and Stress Research Group, pp. , 2011.

[45] T. Lanciano, and V. L. Zammuner, "Individual differences in work-related well-being: The role of attachment style", Europe's Journal of Psychology, vol. 10, no. 4, pp. 694-711, 2014.

[46] G. B. Hall, M. F. Dollard, A. H. Winefield, C. Dormann, and A. B. Bakker, "Psychosocial safety climate buffers effects of job demands on depression and positive organizational behaviors”, Anxiety, Stress \& Coping, vol. 26, no. 4, pp. 355-377, 2013.

[47] M. F. Dollard, and W. McTernan, "Psychosocial safety climate: A multilevel theory of work stress in the health and community service sector", Epidemiology and Psychiatric Sciences, vol. 2, pp. 1-7, 2011.

[48] A. Seidler, M. Thinschmidt, S. Deckert, F. Then, J. Hegewald, K. Nieuwenhuijsen, and S. G. Riedel-Heller, "The role of psychosocial working conditions on burnout and its core component emotional exhaustion - A systematic 
review”, Journal of Occupational Medicine and Toxicology, vol. 9, no. 10, 2014, http://www.occup-med.com/content

[49] D. Nahrgang, F. P. Morgeson, and D. A. Hofmann, "Safety at work: A meta-analytic investigation of the link between job demands, job resources, burnout, engagement, and safety outcomes”, Journal of Applied Psychology, vol. 96, pp. 71-94, 2010.
[50] K. P. Cigularov, P. Y. Chen, and J. Rosecrance, “The effects of error management climate and safety communication on safety: A multi-level study", Accident Analysis and Prevention, vol. 42, no. 5, pp. 1498-1506, 2010.

[51] S. A. Bond, M. R. Tuckey, and M. F. Dollard, "Psychosocial safety climate, workplace bullying, and symptoms of posttraumatic stress”, Organization Development Journal, vol. 28, pp. 37-56, 2010. 